\title{
A Haptic Virtual Borescope for Visual Engine Inspection Training
}

\author{
Deepak Vembar \\ School of Computing
}

\author{
Andrew T. Duchowski* \\ School of Computing
}

\author{
Sajay Sadasivan \\ Industrial Engineering
}

\author{
Anand K. Gramopadhye \\ Industrial Engineering
}

Clemson University, Clemson, SC 29634

\begin{abstract}
A haptic virtual borescope is developed for the purpose of aircraft engine inspection training, similar in spirit to borescope trainers intended for use in gas turbine maintenance training schools. Such devices consist of engine section mockups for use with a real borescope. Our approach instead simulates engine sections in virtual reality, replacing the need for physical mockups. We model the engine casing as a "black box" where a simulated borescope tip is inserted (in practice a real borescope is used to provide tactile veridicality of the probe's braided sheath but the camera at its tip is not used). The probe's translational movement is mapped to the virtual camera's. The graphical engine representation can conceivably generalize to any engine section that can be modeled graphically. Since the interior chamber of the "black box" casing is empty, the critical component of our simulator is correct borescope tip navigation as well as force feedback response based on a mathematical model of collision detection of the tip in the computer generated environment.

Haptic response is thought to be a key component of the simulator as it provides non-visual tactile awareness of the borescope tip within the engine under inspection and, more importantly, its contact with engine surfaces. Our contribution is two-fold. First, we design a novel motor-powered clamp that provides collision response to collision of the camera detected in virtual space. Second, we attempt to isolate the effect of the system's tactile response and provide empirical evaluation of its utility. In line with previous results, our empirical analysis reveals a trend toward a benefit in performance (speed), but suggests that the provision of haptic feedback, while preferred over a solely visual interface, may be perceived as extraneous in a visually-dominated discrimination task.
\end{abstract}

Index Terms: I.3.6 [Computer Graphics]: Methodology and Techniques-Ergonomics; J.4 [Computer Applications]: Social and Behavioral Sciences-Psychology.

\section{INTRODUCTION}

Borescope inspection in the aviation field is an essential nondestructive inspection (NDI) procedure used to examine aircraft components for defects. It is usually performed in an area or enclosure that is not easily accessible without a complete tear-down of the components. Inspection of an aircraft engine, for example, is a common borescope task performed to prevent potentially costly dismantling of the engine. To promote novice inspectors' adoption of inspection strategies exhibited by experienced inspectors, we have built a training simulator for teaching good practices and skill sets associated with the borescope inspection task. This is radically different from on the job training currently adopted, where the novice inspector serves as an apprentice to an experienced inspector on the hangar floor. Although several variants of the borescope are used in practice, in this paper we virtualize the video borescope: an

*e-mail:duchowski@acm.org

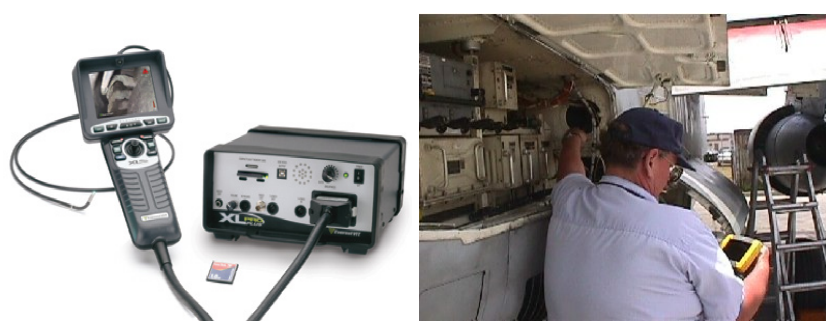

Figure 1: Video borescope and the inspection procedure.

instrument with a flexible fiber-optic probe that uses a video screen for image output (a popular and cheaper alternative is the optical borescope that uses an eyepiece in place of a video screen).

The video borescope (Figure 1) consists of a base unit with an attached monitor, a flexible fiber-optic probe with a CCD camera and a hand-held interface for controlling the articulation of the probe tip. Similar devices, called endoscopes, have been used in the medical field for surgical path planning and minimally invasive surgical procedures. The base unit consists of a light source, a video processing unit to manipulate the video feed from the camera, and memory card slots for storing captured images.

The probe consists of a magnifying CCD camera attached to the probe tip that transmits the images through the optical fibers to the base unit. Optic fiber is used to transmit light from the base unit to illuminate the internal components being inspected. The probe tip can be articulated with the control interface, and can rotate almost 300 degrees about its pivot. The entire probe is enclosed in a braided sheath to prevent damage to the fragile optical fiber. A key concern during operation is damage to the probe tip. Tip replacement is expensive and so training for prevention of equipment breakage is worthwhile.

The manual interface is a light, one-handed device possessing a mini-joystick that is used to control the articulation of the probe tip. Buttons are also available to allow the inspector to take screen captures of the video, record live video for off-line analysis, freeze the articulation of the probe, and compare the size of the defects encountered during the inspection process.

In addition to the visual feedback provided by the CCD images, the borescope inspector also feels force feedback when the probe collides with the engine components. Unlike similar devices used in the medical field, the collision is between the semi-flexible probe and a rigid body. Hence there are no elastic forces exerting feedback on the borescope, unlike tissue and organs in medical endoscope simulators. For simplicity, the forces experienced can be broken down into contact forces that act on the tip of the probe and sliding forces acting on the probe as it snakes through the engine.

The angle of the probe tip's contact with the rigid surface plays an important role in determining the forces experienced by the inspector. Upon contact with a rigid surface, the tip can either slide, stop, or deform. When the angle of incidence between the tip and the contact surface is large, the tip will ether slide along the surface unless it encounters further hindrance or it will stop and deform if its motion is impeded. When the angle of incidence of the 
borescope tip is almost perpendicular, the probe may deform if the force applied by the inspector is large (e.g., when s/he does not realize the tip has hit a surface). The probe's deformation will depend on the physical characteristics of the probe and the contact surface. If the force applied by the inspector is not as large (e.g., the inspector stopped pushing the probe due to visual feedback), no deformation will occur and the tip will stop.

The borescope inspection procedure is an example of a visual search and discrimination task. The primary aim of the procedure is to look for engine component anomalies or defects. The search aspect of the task can be broken down into navigation of the borescope through the engine, articulation of the camera to acquire a useful field of view, and a visual search within the given field of view to locate defects. Once a defect is detected, the task shifts from search to discrimination. Based on prior experience or standardized defect categories, the inspector has to decide whether the severity of the defect warrants corrective action. Experienced inspectors usually follow a systematic search strategy in the inspection procedure [14]. If they are inspecting a turbine, they first inspect the leading edge of the blade from the base of the blade to the tip, then follow along the front face of the blade to the trailing edge (see Figure 2). The trailing edge is then inspected from the tip to the base of the

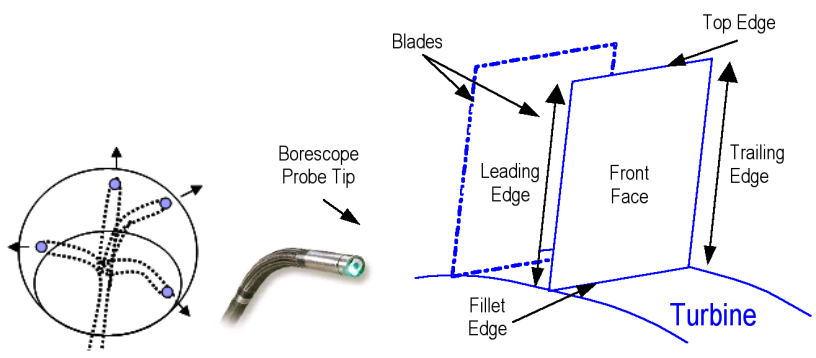

Figure 2: The video borescope's articulating tip and the turbine inspection process.

blade and back to the front of the blade. Thus the inspection procedure follows a systematic, circular motion by which the inspector can detect and identify defects present on the blade. The inspector then moves to the next blade of the turbine and repeats the procedure. Once all the front faces of the blades are inspected, the inspector changes the orientation of the probe to face the back face of the blade. The same systematic search procedure is repeated for the back faces. Any defects found during inspection are logged and the engine is dismantled for further repairs if necessary.

Borescopes have been in use by aircraft engine mechanics for many years, but the training required to operate and master these tools is expensive and lacks standardization. While there are standards and regulations that govern aircraft maintenance in general, none provide detailed information on borescope training. The American Society for Nondestructive Testing (ASNT) is an organization that is recognized for its leading role in maintaining regulations for NDI training. Currently, the ASNT only provides a brief introduction to borescopes in levels I and II of their visual testing curriculum [1]. While the ASNT requires a written examination, a hands-on test, and experience to become qualified for NDI inspection, it does not provide any standards by which borescope inspectors can become qualified. The FAA engine certification program, which details individual engine certification requirements for aircraft engine technicians, only refers to engine manufacturers' instructions for borescope usage [14]. Although this organization provides a general overview of borescope use, it lacks detail and standardization in the area of borescope inspection training.

To facilitate inspection training, we have built a haptic virtual borescope. The simulator relies on visually realistic geometric models of the aircraft engine, customizable textures of defects, and active haptics to provide force feedback to the user. First, we developed software for virtual borescope navigation within functionally realistic graphical models of an engine. We evaluated this functionality in desktop, non-immersive environments and then built physical components to resemble a real video borescope. Navigation of the borescope's articulating tip is simulated by feeding a real borescope's braided sheath through a newly constructed device providing haptic feedback in reaction to the tip's physical interaction with virtual objects (e.g., engine stator and turbine fan blades). In this paper we concentrate our evaluation on the efficacy of this force feedback in terms of usage performance.

\section{BACKGROUND}

Virtual reality (VR) simulators have been used as adjuncts to conventional learning in a variety of fields from medical training to manufacturing and design applications. The simulators provide a realistic, real-time environment with the ability to provide instantaneous feedback on performance. Training sessions can be easily customized to suit individual needs and skill levels. Prior research has shown that the skills training obtained from the use of simulators is similar to those obtained from real-world experiences and that these skills successfully transfer to task performance $[4,13]$. However, the fidelity of the VR simulation is crucial for the skills to transfer effectively from the virtual testbed to the real world.

Training simulators have mainly been visual in nature. Haptic devices such as SenseAble Technologies' PHANToM have made it easy to incorporate force feedback into virtual models. Prior studies have shown that haptic feedback plays an important role in improving performance [17], augmenting the skills transfer in novice trainees, and that early exposure to force feedback in the simulator improves performance [15]. In the presence of multiple input modalities including visual, auditory, and haptic, intermodal integration may be a key psychological mechanism contributing to the sense of presence in the virtual environment. While it is reasonable to assume that the simulator with the highest haptic fidelity will be perceived by the user as the "most real" interface, psychophysical testing on human subjects is needed to reveal the minimum performance requirements of the hardware interface to be used in the simulator.

One of the simplest forms of haptic feedback is the interaction of a rigid body with a rigid manipulator. An analysis of human perception of a rigid surface revealed that the perception of encountering a rigid surface is not as strongly correlated to the stiffness as it is correlated to the initial contact with the surface [12]. Furthermore, when using a haptic interface to simulate contact forces, Tan, et al. [2] showed that users have poor force direction discrimination resolution. This suggests that although users can perceive the magnitude of the force, they are unable to perceive the direction of the simulated force with the same resolution.

Recent studies of haptic interfaces have focused on the use of a commercial interface (PHANToM) when used in assembly tasks where a hand-held object is being manipulated (e.g., peg-in-hole task) [7, 8, 3]. As a first step toward developing a haptic force feedback device for the borescope, we developed a simple OFF/ON haptic device using a cheap off-the-shelf motor kit. O'Malley and Upperman have discussed human performance in size identification and size discrimination tasks with the PHANToM [10]. Our custom research-quality haptic device is more suitable for the borescope inspection task since it allows inspectors to physically insert the probe into a simulated engine just as they would in the real situation. We are not aware of any other existing virtual borescope simulator comparable to ours. Furthermore, our task differs from the peg-in-hole assembly task in that the user does not have the benefit of seeing the borescope (e.g., peg), rather, the user is the peg, as it were, and sees the virtual environment from the peg tip's point of view. 

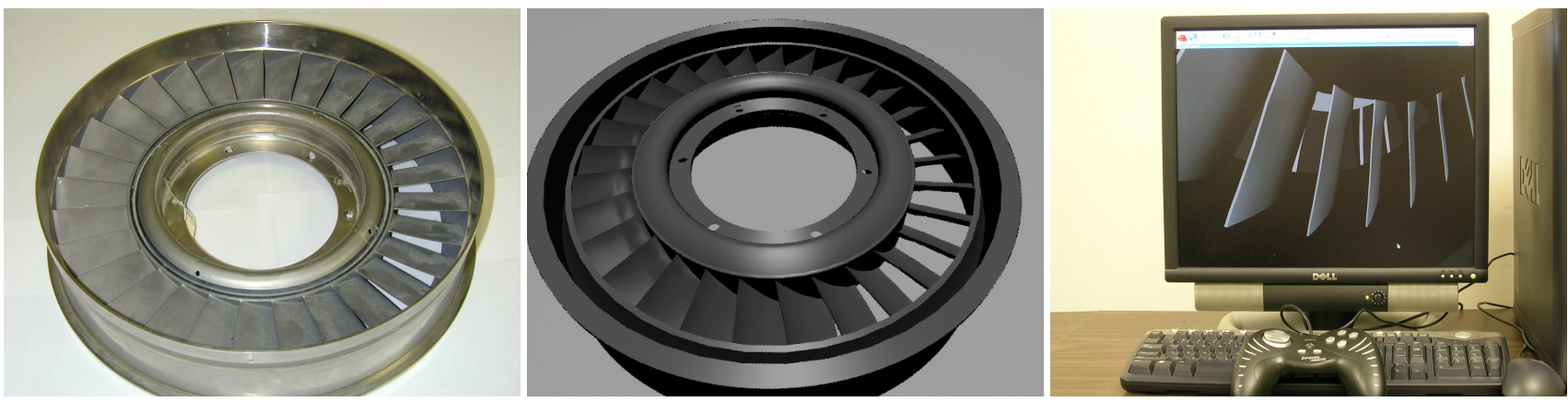

Figure 3: Actual and modeled engine sections (left, middle), desktop prototype of borescope navigation (right).

\section{BORESCOPE INSPECTION SIMULATOR}

Since our borescope is aimed at engine inspection, our first task was construction of a suitable geometric model for the inspection test scenario. We modeled one stage of the hot section of the aircraft engine in $\mathrm{Maya}^{1}$ and exported it as an Alias|Wavefront . obj file with texture and material information. Our simulator renders the models using a custom viewer written in OpenSceneGraph (OSG), ${ }^{2}$ an open source API. The environment consists of an enclosed stator and turbine modeled on the interior of a real engine (see Figure 3). The model is texture-mapped and lit to simulate the visual look and feel of an actual borescope camera image. Figure 4 shows a screenshot from the simulator compared to an actual video borescope frame.
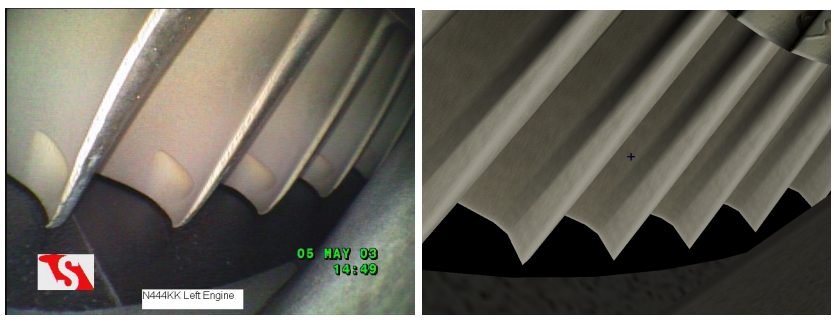

Figure 4: Visual output from the actual borescope (left) and our simulator (right).

The translation and rotation of the camera are controlled by the relative change in the mouse coordinates obtained from the the first stage of the haptic box (see below), which in turn is controlled by the participant inserting or withdrawing an actual probe into a guide tube fixed within the haptic box. The translation of the camera is always along the view vector, either forward when the user pushes the probe into the haptic box or in reverse when the probe is withdrawn.

A simple collision response is implemented in the simulator. At every frame, changes in camera position and orientation are checked with the bounding sphere of the nearest component in the virtual model. The camera is updated only if there are no penetrations of the surrounding model. Note that this does not take into consideration the angle of incidence of the camera with the intersecting surface. In the real borescope, the behavior of the probe is dependent not only on the angle of contact with the surface, but also the force applied by the user and the location of the probe tip.

Following development of the borescope simulator's visual models and basic 3D navigation, we focused on three main interactive tasks involved in the inspection process.

\footnotetext{
${ }^{1}<\mathrm{http}: / /$ www.autodesk.com/fo-products-maya/>, last accessed 01/08.

${ }^{2}<$ http://www.openscenegraph.org/ $>$, last accessed 01/08.
}

1. Probe feed. The probe's position and orientation is governed by its motion constrained within 2 degrees of freedom. It can be manually pushed into or pulled out of the engine, combined with its roll about its longitudinal axis. In/out translation of the probe determines its location within the engine, while its axial rotation determines the camera's attitude. Although the probe is flexible, the amount of bend is constrained by the bundle of fiber-optic cables and its braided sheath.

2. Camera articulation. The CCD camera's articulation is controlled by manual (hand-held) joystick controls. The operating region of the probe tip forms a partial sphere and has two degrees of articulation, yaw and pitch, about the local $x$ and $y$ axes (see Figure 2). In our implementation, an unconstrained camera model is used to simulate the borescope camera. This model does not take into consideration the limited motion of the physical camera and allows for a full $360^{\circ}$ rotation about its axes. Camera control is provided by an off-the-shelf Logitech USB gamepad. The analog D-pad is used to control the rotation of the camera along the $x$ and $y$ axes simulating real borescope controls.

3. Visual and force feedback. Visual feedback on the monitor is the borescope's primary form of feedback. In addition, the inspector also receives force feedback whenever the tip of the probe collides with the rigid engine components. The real probe may drag along the surface of the engine, it may stop, or it may become entangled in the blades.

In this paper we concentrate on the development and evaluation of a simple force feedback interface to the borescope simulator. Task analysis and evaluation of the visual fidelity of the geometric engine model and rendering have been reported elsewhere [16].

\subsection{Design of The Haptic Interface}

Off-the-shelf devices such as the PHANToM [9] simulate contact forces with a high degree of realism, but are expensive and are not suitable for all applications. Since we are developing simulators that will be used in the classroom for training students, we wanted to find interfaces that are cheap, sturdy, and easy to maintain. The primary requirements of the borescope interface are simulation of the probe feed to increase the behavioral realism of the simulator and provision of simple, synchronous force feedback to the user based on intersections of the virtual camera with the engine model.

Figure 5 shows a top-down view of the haptic box designed to address the main requirements of the simulator. The first stage of the device consists of an entry point that guides the probe beneath an optical mouse, as the probe enters the box from the left side (the haptic component has been removed to show the guide tube into which the probe is inserted). The dimensions of the wooden enclosure are $6^{\prime \prime} \times 6^{\prime \prime} \times 24^{\prime \prime}$. The aluminum guide tube is $1 \mathrm{~cm}$ in 


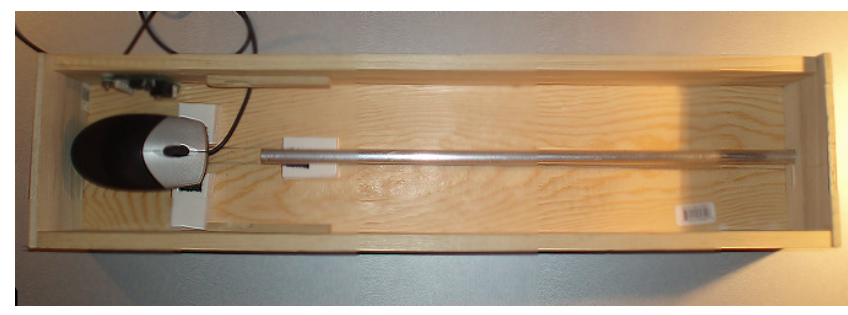

Figure 5: Top view of the feedback device with mouse and guide tube, with probe entry at left (the haptic component has been removed to show the guide tube into which the probe is inserted).

diameter and runs through the entire length of the box, ensuring that the probe follows a predefined path within the enclosure. The box was designed for a borescope probe $8 \mathrm{~mm}$ in diameter, but can be used for any probe thickness by changing the guide tube's diameter.

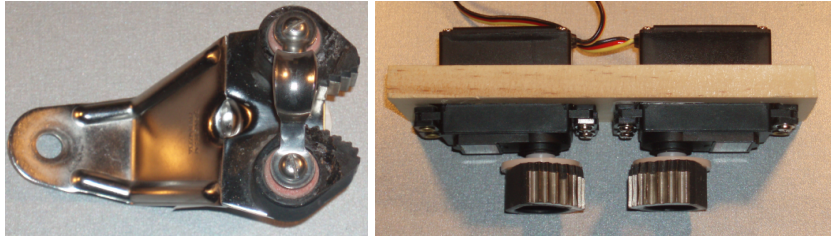

Figure 6: Sailing cam cleat with spring-loaded cams (Ronstan, Australia) prior to its refurbishment for the simulator (left); following its attachment to the servo motors (right).

The second stage of the haptic box contains the force feedback device consisting of two servo motors attached to two serrated cams obtained from a cam cleat formerly used in its original sailing application (Figure 6). A cam cleat is normally used to maintain sail tension by preventing a sailing control line (e.g., main sheet) from backing up through the cleat. In its present configuration, it is used in the reverse, preventing a line (the borescope probe) from being inserted forward. To allow computer-controlled operation of the cam cleat, its springs were removed and its cams made operable via attachment to the two servo motors (see below). The main components of the haptic interface follow.

1. Optical mouse. The mouse provides the measurement of probe feed (mouse $\Delta y$ ) and probe twist (mouse $\Delta x$ ) as it passes through the guide tube. A 4 " section of the tube is glued to the underbelly of the mouse with a small notch cut out to allow the optical sensor of the mouse to detect motion as the probe moves through the tube. A scaling factor, calculated from the physical diameter of the probe and the relative $\Delta x$ changes in the mouse coordinates, is used to provide accurate visual feedback when the user twists the probe (e.g., with the probe approx. $2.5 \mathrm{~cm}$ in circumference, mapping to 350 pixels when using the mouse, one complete rotation $(2 \pi=350$ pixels) of each pixel change is about 0.018 radians, the camera roll scaling factor is therefore set to 0.02 ).

2. Servo motors and the Phidgets [5] interface kit (Figure 6). We used two servo motors mounted on a wooden platform as the primary force feedback interface. The motors are mounted such that the probe passes midway between the two motors after it exits the guide tube in the first stage. The servos operate at $50 \mathrm{~Hz}$ with an accuracy of $0.1^{\circ}$. An interface kit mounted along the inside wall of the enclosure controls both the servos. The interface kit is connected to the computer through USB and allows the simulation program to read and set the values of the motors.
3. Cam cleats. The re-configured cam cleats are glued to the servo motors with the help of attachments that were provided in the motor kit.

4. Aluminum tube and probe. A $1 \mathrm{~cm}$ diameter aluminum tube is used along the base of the box to guide the probe through the box. The length of the guide tube beyond the motors is approximately $18^{\prime \prime}$. The guide tubes were cut to size and placed in the box such that the probe is always in the guide tube, except when it passes though the cam cleat. The probe used was that of a real but inoperative optical borescope.

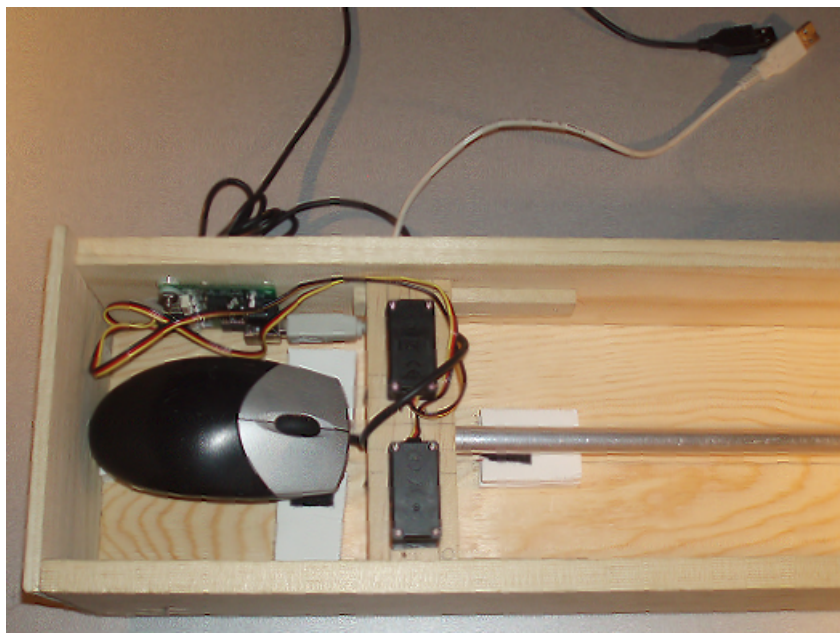

Figure 7: Haptic feedback interface with motors installed.

\subsection{Operation of Haptic Interface}

The haptic interface can be divided into two related functions: probe feed measurement provided by the optical mouse and active force feedback provided by the Phidgets interface unit. In the first stage of the interface, the borescope probe passes underneath the optical mouse. The braided cable enclosing the optical fibers of the borescope provides a sufficiently rough surface to enable the optical sensors of the mouse to detect motion. The guide tube attached to the base of the mouse constrains the motion of the probe such that the optical sensors can pick up slight changes in the probe's translation and twist.

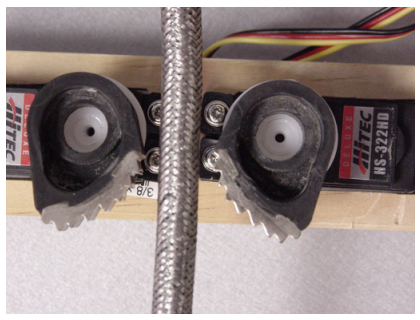

(a) Probe free with cams open.

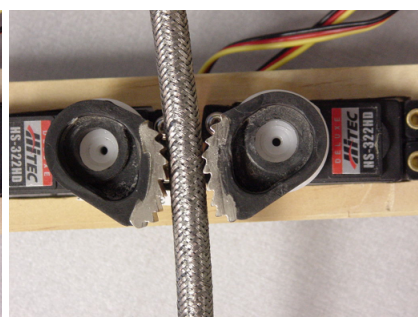

(b) Probe clamped by cams.
Figure 8: Motor operation with the motorized cam cleat (direction of insertion is up). Note that once clamped, further insertion is prevented, however, retraction of the probe is still possible.

The interaction of the motors with the simulation is more involved. The cam cleat was specifically chosen because of its serrated cams. Figure 8 depicts the two stages of the motor operation. In Figure 8 (a), the position of the two motors is such that the cams are open and the probe is free to pass through (in both forward and 
reverse directions). Figure 8 (b) shows the position of the two motors that results in the cams clamping shut on the probe. The force applied to the probe by the cams is just sufficient to prevent the probe from moving in the forward direction but provides no hindrance to translation in the reverse direction. The serrated metal cams grip the probe's sheath and provide a slip-free hold on the probe.

The servo motors are controlled with the Phidget motor interface board. This board is connected to the computer through the USB interface and is integrated into the virtual borescope simulator. When there is no intersection of the virtual camera with the model geometry, the servo motors are programmed to open the cams to allow free motion of the probe. When the simulation detects intersection between the virtual camera and the model geometry, the motors rotate the cams shut. Note that we use only one pair of motors to simulate force feedback in only one (the forward) direction.

\section{Empirical Evaluation}

The purpose of the empirical evaluation was to gauge the effectiveness of the haptic force feedback provided to probe users. We hypothesized that haptic feedback would provide a measurable effect in simulator usage. We were also interested in users' subjective impressions of the device.

\subsection{Experimental Design}

A within-subjects design was used for this experiment due to the limited pool of experienced borescope inspectors. We tested the simulator under two conditions: visual only condition $(V)$ and combined visual and haptic condition $(V+H)$. Haptic feedback constituted the independent variable. Time taken to complete inspection of the virtual model served as the dependent variable. The alternating order of the two conditions was balanced evenly between subjects so that half started with the visual only condition first while the other half started with the combined condition.

\subsection{Subjects}

Eight participants (all male) were recruited to evaluate the virtual borescope and the force feedback device. All participants were familiar with optical borescope inspection and had prior experience in aircraft engine inspection (average experience was about 6 years). The aims of the experiment were explained to all participants and informed consent and demographic data collected prior to its commencement. All participants were right-handed and were seated during the testing phase of the experiment (Figure 9).

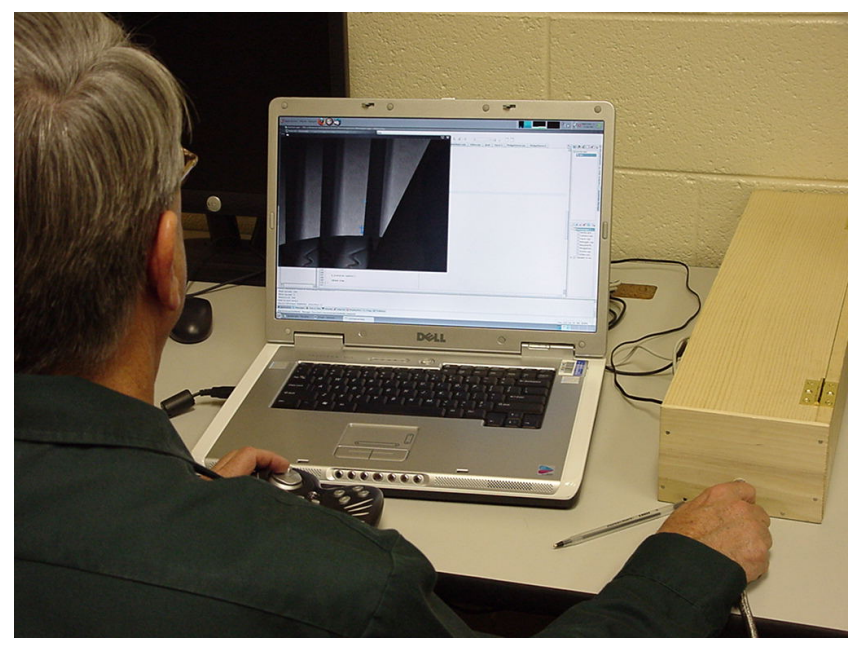

Figure 9: Experimental setup.

\subsection{Apparatus and Stimulus}

The simulator was run on a Dell 9300 laptop, equipped with 2 GB RAM and a GeForce 6800 video card. The simulator's visual output was presented in a $1024 \times 768$ window on the $17^{\prime \prime}$ screen on the laptop. The simulator maintained an interactive frame rate $(60$ fps) throughout the experiment. The laptop was placed on the table directly in front of the participant.

The haptic box was placed about $6^{\prime \prime}$ to the right of the laptop so that the participants used their dominant hand for the probe feed, and their non-dominant hand to control the gamepad. An old borescope probe, approximately 6 feet in length was manually inserted by the volunteers into the haptic box during the experiment. To simulate the rotation of the turbine, one of the gamepad buttons was mapped to modify the scene graph to rotate the turbine (in the real task, turbine rotation is usually performed manually by the inspector or by an accomplice). The participant can visually observe the turbine rotating when the button is pressed. The button was selected such that the participant could control the manipulation of the virtual camera with one hand on the gamepad.

In both test conditions, participants used the gamepad and the probe feed to control the orientation and translation of the virtual camera. The visual feedback that the participants received was the same in both conditions. The only difference was the feedback that the participants received when the software detected intersection of the virtual camera with the engine model geometry. In the visual only condition $(V)$, the only indicator of camera intersection was visual, i.e., the simulator would not update the display until the participant either changed the position of the camera by moving the probe through the haptic box or changed the orientation of the camera. In the visual and haptic condition $(V+H)$, intersections of the virtual camera with the model in the forward direction resulted in the motors engaging the cam cleat to clamp on the probe. The forces on the probe were calibrated such that it would not move in the forward direction but could be pulled out easily. The clamps disengaged when the participant either pulled out the probe or changed the orientation of the virtual camera and there was no penetration of the camera with the model.

Due to the use of the servo motors to control the force feedback, it was observed that engaging and disengaging of the cam cleats was accompanied by an audible noise from the motor. Prior research has shown that auditory cues can influence task performance, either when separate from visual cues or when used concurrently with visual feedback [19]. To prevent the participant from using these auditory cues, the device was completely enclosed to dampen the motor noise. To further mask the cam cleat's motor noise, a dummy motor was placed in the haptic box which randomly changed its position and emitted a sound similar to the motors controlling the cam cleats. Participants were informed that the noise was the probe feed device working. The participants were intentionally kept unaware of the contents of the box.

\subsection{Procedure}

To begin, participants filled out a brief inspection experience questionnaire. They were then seated in front of the test computer and were instructed on the use of the gamepad to control the orientation of the virtual camera and the probe feed to control the position of the camera. The starting position of all the participants was the same. Participants were instructed to maneuver the camera through the first stage of the stator and start the inspection of the rotor, as they would normally do in the real task (see Figure 10).

Instead of defects in the model of the engine blades, visual markers in the form of a numbered sequence were used to guide the inspection process. The participants were instructed to first inspect the leading edge of the blade and then the trailing edge. On completion of one face, they were asked to change the orientation of the camera to inspect the back face of the next blade. Once they had 

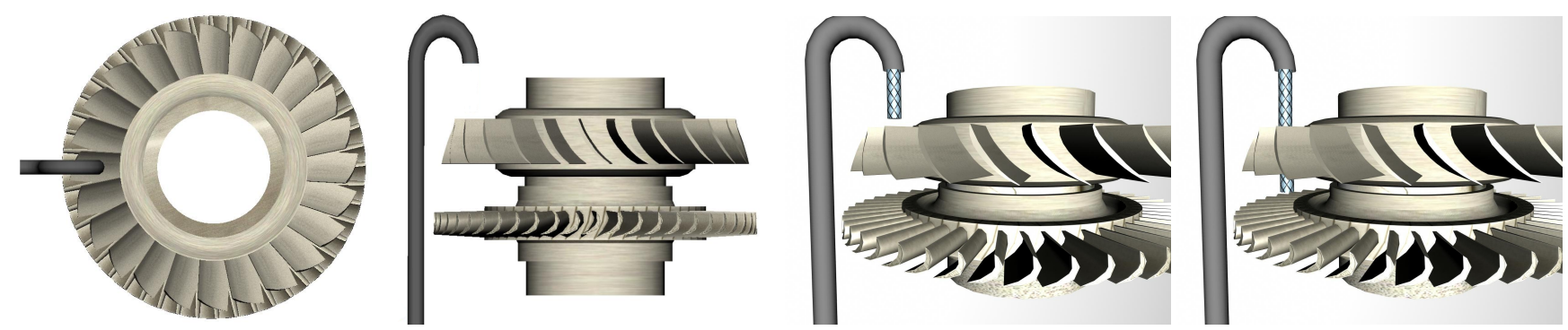

Figure 10: Inspection scenario (from left to right): top and side views inside the engine casing, with guide tube exit positioned just above the stator; braided textured cylinder illustrates the typical path of the simulated borescope through the stator to its position during turbine (rotor) blade inspection. Once in position, the task mainly consists of rotating the turbine to visually inspect each of its blades. Note that the user never sees this external viewpoint-the user's only viewpoint is from the tip of the borescope.

completed inspection of one blade, participants were asked to move onto the next blade by pressing the gamepad button to turn the rotor and repeat the inspection process.

In the first phase, participants were provided training with the visual stimulus and the haptic box. This phase was untimed and allowed familiarization of the control interfaces. Once users were proficient in using the interfaces, they were asked to inspect the first 5 marked blades on the rotor under one of $V$ or $V+H$ conditions. On completion of this first task, participants were given post-test and workload questionnaires to complete. After a brief familiarization phase with the second interface, participants performed the same inspection process with the second interface. Participants then filled out a second questionnaire followed by a post-test debriefing.

\subsection{Data Collection}

In addition to the subjective data collected in the form of questionnaire responses, we also collected performance data in terms of time taken to complete each of the tasks. The questionnaire was divided into three sections: visual realism, interface evaluation, and perceived workload. The visual and interface related questions were derived from Witmer and Singer's presence questionnaire [18], while the workload section was derived from the NASA TLX questionnaire [6]. The responses for the all the questions were on a 5-point Likert scale, with 1 indicating strong disagreement, 5 strong agreement, and 3 neutrality.

\section{Results}

Performance data collected was time taken for completion of the task. The average time taken for completion of the task with both visual and haptic feedback $(V+H$ treatment condition; mean time $=$ $280.75 \mathrm{~s}$ ) was shorter than the average time taken to complete the task with only visual feedback ( $V$ condition; mean time $=306.50$ $\mathrm{s})$, although not significantly so $(\mathrm{F}(1,7)=0.58, \mathrm{p}=0.47$, n.s.), according to a repeated measures one-way ANOVA. An ANOVA performed on the completion times with the participants as the blocking factor revealed the participant as a significant main effect $(\mathrm{F}(7,7)=5.56, \mathrm{p}<0.05)$, as shown in Figure 11, indicating significant variability between subjects.

Friedman's test performed on the subjective data found significant difference in only one of the questionnaire responses (Q11; $\chi^{2}(1, N=8)=3.84, \mathrm{p}<0.05$, see Table 1$)$. Figure 12 shows the average responses to the questions provided by the participants. In the post-test debriefing, 6 out of 8 participants preferred the simulator with force feedback, one had no preference for either condition while the last had no comments.

\section{Discussion}

Results indicate no significant effect of haptics on performance. The data suggest a trend of participants completing the task faster
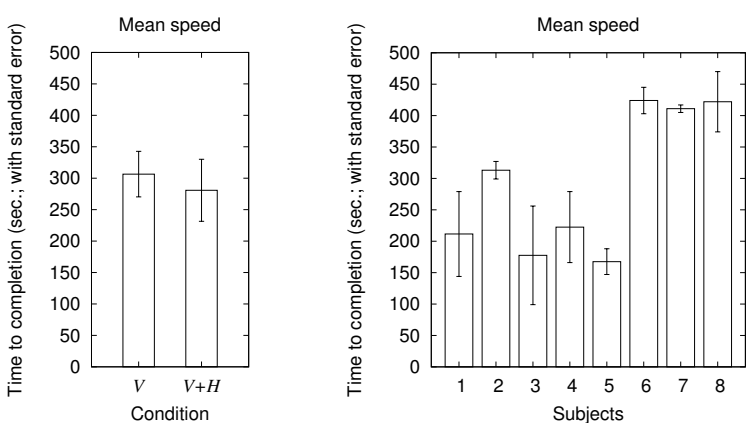

Figure 11: Speed comparison between conditions and subjects: $\mathrm{Vi}-$ sual $(V)$, Visual and Haptic $(V+H)$.

with haptic feedback, but the significant variability among subjects may be masking its effect on performance. The results, although inconclusive, are in line with previous studies suggesting that haptic feedback improves performance (mean time to task completion) $[15,17]$. Lack of significance in our performance analysis may be due to the inspectors' inexperience with a video borescope or the simplicity of our chosen task, as explained below.

Participants in our study were experienced borescope inspectors, but their inspection experience was mainly with the optical borescope and not the video borescope we are simulating. Of the 8 participants, only 2 had prior experience with the video borescope, while the remaining 6 had limited to no previous experience. Unlike its video counterpart, the optical borescope has simpler probe articulation controls and an eyepiece for visual feedback from the probe's tip that is much smaller than the video borescope's screen. We suspect that participants paid more attention to the visual output due to its novelty, i.e., its larger field of view and higher resolution than what they were accustomed to. Furthermore, because vision tends to dominate touch (particularly when in conflict [11]), and because the inspection task is inherently visual, it may be that haptic feedback was simply ignored under present experimental conditions (e.g., the task lacked a sufficiently compelling tactile component to begin with).

Engine inspection is a repetitive process, where the inspector first maneuvers the probe through the guide tube past numerous internal components of the aircraft engine. Once the blades to be inspected are in the borescope camera's field-of-view, the interaction is limited to the articulation of the probe tip and hence the camera. Instead of translating the probe, the inspector rotates the blades to inspect the rest of the engine. Tactile feedback is not needed for this task, rather, it is only needed when the inspector is maneuvering the probe into and out of the engine-we conjecture this is when tactile 
Visual section

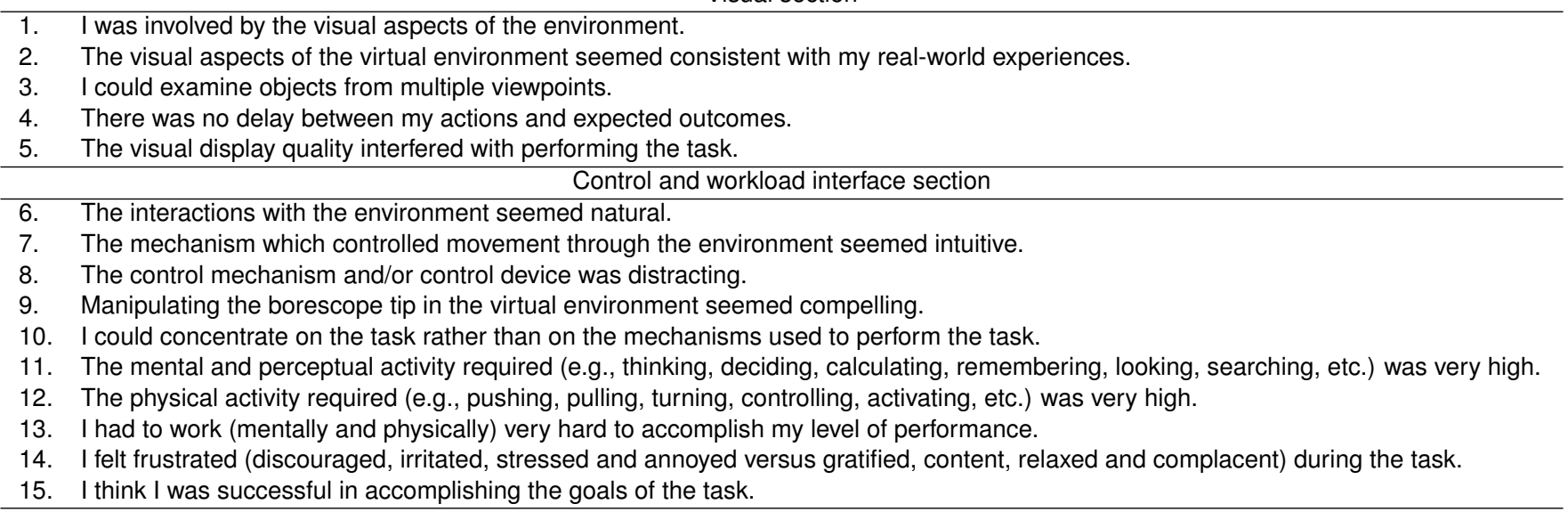

Table 1: Subjective questionnaire.

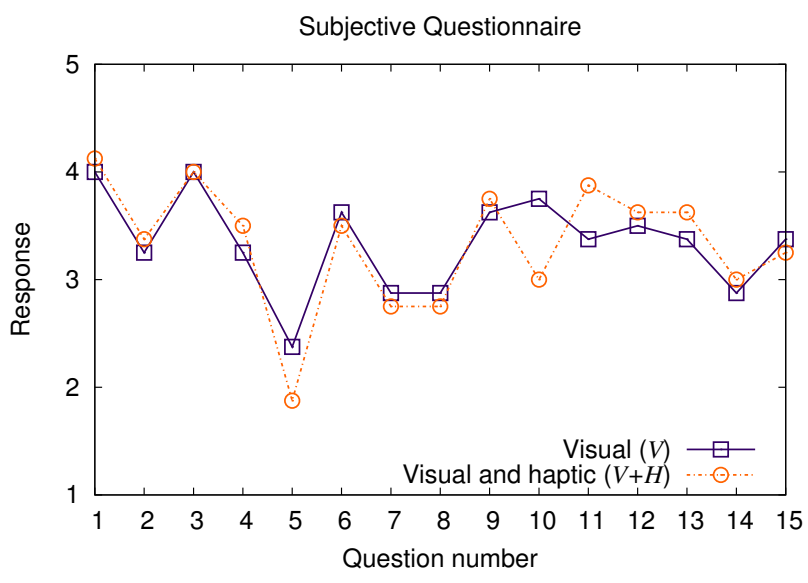

Figure 12: Subjective impressions (average) regarding system use. Users' responses were made on a 5-point Likert scale corresponding to agreement with the questionnaire statements (1:strongly disagree, ..., 5:strongly agree) listed in Table 1.

response is most important for avoiding damage to the borescope camera. In our experiment, participants were given a very simple scenario where they navigated through just one set of stator blades and performed a limited inspection of 5 blades of the turbine rotor. As the inspection was performed mainly by changing the orientation of the camera through the gamepad controls, haptic feedback was of little use during the actual visual inspection process.

The responses of the participants to the questionnaire revealed that they perceived the visual stimulus to be somewhat similar to that of the actual task (Q2). Since the visual stimulus was the same in both experimental conditions, no significant difference conditions was detected (none was expected). Participants reported that they were involved in the task by the visual output of the simulator (Q1) and that they could examine the objects from multiple viewpoints $(\mathrm{Q} 3)$. From the remaining responses concerning visual realism (Q4 \& Q5), we can infer that the interaction delay and visual display characteristics of the simulator did not significantly alter the perception of the inspection process. During the post-test debriefing, participants commented on the visual realism of the simulator, stating that it conveyed the engine inspection process accurately.

In the interface evaluation part of the questionnaire, participants noted that the interaction with the environment seemed somewhat natural (Q6). In the debrief some commented that they became "as lost in this as the real thing" and most noted that they had trouble getting used to the single-handed gamepad interface (although their response regarding the intuitiveness of the control (Q7) was neutral). Unlike the real control interface, we chose to use an offthe-shelf gamepad, which is normally manipulated by both hands. Since participants had to use their dominant hand to control the probe feed, they had to use their non-dominant hand to balance and control the gamepad. Also, most of the participants had experience with the optical borescope where the control interface is simpler than the video borescope's. In this experiment, since the gamepad was used equally in both conditions, we can infer that it did not have any effect on performance. The neutral response to the question concerning distraction of the control (Q8) supports this reasoning.

The workload effects portion of the questionnaire compared the experience of using the virtual simulator under the two conditions. Participants reported that the mental and perceptual activity required for the inspection task was significantly higher with the haptic force feedback than without (Q11). This suggests that participants may have found the provision of haptic feedback extraneous in this particular task. Given the simplistic nature of the task, and the limited opportunity for interaction of the probe tip with the model surfaces, we believe that vision dominated the test scenario. The addition of haptic feedback may have distracted participants (hampered their concentration; Q10) and required marginally greater perceived mental and physical effort (Q12 \& Q13).

Most participants reported in the debrief that the simulated representation of the engine interior was visually realistic. However, they found the unconstrained articulation of the camera not to be as realistic as the actual optical borescope's. They reported that unlike the actual borescope, they could perform a full rotation of the camera. Participants also mentioned that the control interface took practice getting used to, but once they had experience using the articulation controls, they felt proficient in using the gamepad. At the end of the experiment, most participants noted that they preferred the provision of haptic response over the purely visual interface. Some noted that the combined interface "feels like the real thing" and "you need to have some force when the borescope hits the engine". Overall they reported positive experience with the haptic interface and one suggested that, for training purposes, intensive training with the visual only condition for familiarization with the control interface followed by simulator experience with haptic feedback would provide the best learning opportunity. 


\section{Conclusion \& Future Work}

We have presented the development and evaluation of a simple haptic interface for borescope inspection. The interface is unique in that it is simple to construct and is composed of readily available components that cost less than specialized force feedback devices (e.g., the PHANToM). Performance evaluation of the haptic response component suggests potential for benefit in performance (speed), in line with previous results $[17,15]$. Subjective evaluation revealed that the majority of users preferred the combination of haptic and visual feedback over a solely visual interface even though they may have found the haptic response somewhat extraneous in the present test scenario. Subjective responses also indicated that participants' perceived experiences and workload were not dissimilar from those of the actual inspection process.

The development of the haptic virtual simulator stands to impact current practices by engine inspection personnel using borescopes to conduct inspection during maintenance. The ephemeral quality of the virtual environment makes our simulator applicable to any inspection task where a borescope is needed, e.g., aircraft or shipboard engines.

Future enhancements to the simulator will include building custom interfaces for controlling the articulation of the camera. An updated probe model, including the physical properties of the probe for increased behavioral realism and constraints on the camera motion are planned. Realistic defect textures modeled on the defects commonly found in the engines will be created and the geometry texture mapped to increase the visual fidelity of the simulator. Human performance studies to determine the trade-offs between visual, haptic, and auditory feedback are planned to determine the best metrics needed for accurate inspection training.

Estimating the minimum performance requirements of the simulated interface will require comparison with the real task. Presently we were only interested in isolating the effects of the system's tactile response. In future studies, we intend on increasing the complexity of the task by increasing the number of stators for the probe to snake through. The goal is to match the complexity of the real world task and then to evaluate the utility of the simulator via empirical training transfer experiments.

Note that haptic feedback is currently provided only when the camera intersects the model geometry in the forward direction. Intersections of the camera when the probe is being withdrawn from the haptic box do not result in any force feedback, although this is somewhat important in the actual task. The borescope tip may break if the articulated camera tip catches on a surface during extraction. The reason for the lack of force feedback in our simulator during extraction is twofold. First, the majority of probe maneuvering takes place when the participant starts the inspection procedure. Once the probe is positioned within the space between the two stator blades and the simulated engine inspection has begun, the primary motion is forward probe feed into the box accompanied by camera articulation. Second, in the actual task, the inspector has the option to reset camera articulation by pressing a "home" or reset button. This reduces the chances of probe entanglement and tip breakage when being withdrawn. We therefore felt that at the present time the most important probe contact forces that needed to be simulated were forces in the forward direction. However, an improved probe tip model would allow us to simulate the realistic possibility of tip entanglement during probe withdrawal.

\section{ACKNOWLEDGEMENTS}

The authors would like to thank the volunteers at Stevens Aviation and Greenville Tech Aircraft Maintenance School for their participation. This work is partially funded by DUE grant \#0302780 from the National Science Foundation.

Any opinions, findings, and conclusions or recommendations ex- pressed in this material are those of the authors and do not necessarily reflect the views of the NSF.

\section{REFERENCES}

[1] American Society for Nondestructive Testing. Recommended Practices No. SNT TC-1A, 2001.

[2] F. Barbagli, K. Salisbury, C. Ho, C. Spence, and H. Z. Tan. Haptic Discrimination of Force Direction and the Influence of Visual Information. ACM Transactions on Applied Perception, 3(2):125-135, 2006.

[3] S. Garbaya and U. Zaldivar-Colado. The affect of contact force sensations on user performance in virtual assembly tasks. Virtual Reality, 11(4):287-299, 2007

[4] T. P. Grantcharov, V. B. Kristiansen, J. Bendix, L. Bardram, J. Rosenberg, and P. Funch-Jensen. Randomized clinical trial of virtual reality simulation for laparoscopic skills training. British Journal of Surgery, 91(2):146-150, 2004

[5] S. Greenberg and C. Fitchett. Phidgets: easy development of physical interfaces through physical widgets. In UIST '01: Proceedings of the 14th annual ACM symposium on User Interface Software and Technology (UIST), pages 209-218, New York, NY, 2001. ACM.

[6] S. G. Hart and L. E. Staveland. Development of a multi-dimensional workload rating scale: Results of empirical and theoretical research. In P. A. Hancock and N. Meshkati, editors, Human Mental Workload, pages 139-183. Elsevier, Amsterdam, Holland, 1988.

[7] B. M. Howard and J. M. Vance. Desktop haptic virtual assembly using physically based modelling. Virtual Reality, 11(4):207-215, 2007.

[8] T. Lim, J. M. Ritchie, R. G. Dewar, J. R. Corney, P. Wilkinson, M. Calis, M. Desmulliez, and J.-J. Fang. Factors affecting user performance in haptic assembly. Virtual Reality, 11(4):241-252, 2007.

[9] T. H. Massie and J. K. Salisbury. The PHANToM haptic interface: A device for probing virtual objects. In Proceedings of the ASME Winter Annual Meeting, Symposium on Haptic Interfaces for Virtual Environment and Teleoperator Systems, pages 295-302, 1994.

[10] M. K. O’Malley and G. Upperman. A Study of Perceptual Performance in Haptic Virtual Environments. Journal of Robotics and Mechatronics, 18(4), 2006.

[11] I. Rock and J. Victor. Vision and Touch: An Experimentally Created Conflict between the Two Senses. Science, 143(3606):594-596, 1964.

[12] L. B. Rosenberg and B. D. Adelstein. Perceptual Decomposition of Virtual Haptic Surfaces. In Proceedings of the IEEE 1993 Symposium on Research Frontiers in Virtual Reality, pages 46-53, 1993.

[13] N. E. Seymour, A. G. Gallagher, S. A. Roman, M. K. O’Brien, V. K. Bansal, D. K. Andersen, and R. M. Satava. Virtual Reality Training Improves Operating Room Performance: Results of a Randomized, Double-Blinded Study. Annals of Surgery, 236(4):458-464, 2002.

[14] P. F. Stringfellow, S. Sadasivan, D. Vembar, A. T. Duchowski, and A. K. Gramopadhye. Task Analysis of Video Borescope Operation for Use in a Virtual Training Tool. In Proceedings of Institute of Industrial Engineers Annual Conference, 2005.

[15] P. Ström, L. Hedman, L. Särnå, A. Kjellin, T. Wredmark, and L. Felländer-Tsai. Early Exposure to Haptic Feedback Enhances Performance in Surgical Simulator Training: A Prospective Randomized Crossover Study in Surgical Residents. Surgical Endoscopy, 20(9): 1383-1388, 2006.

[16] D. Vembar, S. Sadasivan, A. T. Duchowski, P. F. Stringfellow, and A. K. Gramopadhye. Design of a Virtual Borescope: A Presence Study. In Proceedings of the 11th International Conference on Human-Computer Interaction, Reno, NV, USA, Jul 2005. Lawrence Erlbaum Associates, Inc.

[17] C. R. Wagner, R. D. Howe, and N. Stylopoulos. The Role of Force Feedback in Surgery: Analysis of Blunt Dissection. In 10th Symposium on Haptic Interfaces for Virtual Environment and Teleoperator Systems, page 73, 2002.

[18] B. G. Witmer and M. J. Singer. Measuring Presence in Virtual Environments: A Presence Questionnaire. Presence, 7(3):225-240, 1998.

[19] Y. Zhang, T. Fernando, H. Xiao, and A. Travis. Evaluation of Auditory and Visual Feedback on Task Performance in a Virtual Assembly Environment. Presence: Teleoperators and Virtual Environments, 15(6):613-626, 2006. 